\title{
Phosphorylation of kinase insert domain receptor by cyclin- dependent kinase 5 at serine 229 is associated with invasive behavior and poor prognosis in prolactin pituitary adenomas
}

\author{
Weiyan $\mathrm{Xie}^{1,2, *}$ Chunhui $\mathrm{Liu}^{2, *}$ Dan $\mathrm{Wu}^{3}$, Zhenye $\mathrm{Li}^{2}$, Chuzhong $\mathrm{Li}^{1,2}$ and Yazhuo \\ Zhang ${ }^{1,2}$ \\ ${ }^{1}$ Beijing Neurosurgical Institute, Capital Medical University, Beijing, China \\ 2 Beijing Tiantan Hospital, Capital Medical University, Beijing, China \\ ${ }^{3}$ Neurological Department, Beijing Renhe Hospital, Beijing, China \\ * These authors have contributed equally to this work
}

Correspondence to: Yazhuo Zhang, email: zyz2004520@yeah.net

Keywords: CDK5; KDR; phosphorylation; prolactinomas; invasiveness; Pathology Section

Received: November 06, 2015 Accepted: June 29, $2016 \quad$ Published: July 12, 2016

\section{ABSTRACT}

Pituitary adenomas constitute $\mathbf{1 5 - 2 0 \%}$ of intracranial neoplasms. Previously we reported that cyclin-dependent kinase 5 (CDK5) is upregulated in pituitary tumors associated with activating protein $\mathrm{p} 35$, and plays an essential role in pituitary adenomas progression. Here we explored the mechanisms of CDK5 signaling in prolactin pituitary adenomas. Our data indicate that p35 expression and CDK5 activity are both significantly increased in human invasive prolactin pituitary adenomas as compared to noninvasive forms of pituitary adenomas. Inhibition of CDK5 activity suppressed cell migration and invasive ability in GH3 rat pituitary cells. We identified that CDK5 phosphorylates serine 229 residue (Ser-229) of kinase insert domain receptor (KDR), also known as VEGFR-2, in prolactin pituitary adenomas. Phosphorylation of Ser-229 is required for proper KDR surface localization. Phosphorylated Ser-229 in KDR (pSer229) levels are significantly higher in noninvasive and invasive prolactin pituitary adenomas compared to normal pituitary tissues. In addition, our data indicated that higher KDR pSer-229 correlates with worse prognosis in patients with prolactin pituitary adenomas. In summary, our results illustrated that CDK5-mediated KDR phosphorylation controls prolactin pituitary adenoma progression and KDR pSer-229 serves as a potential prognostic biomarker for both noninvasive and invasive pituitary adenomas.

\section{INTRODUCTION}

Pituitary adenomas are common neuroendocrine neoplasms arising from adenohypophyseal cells. Although pituitary tumors are typically benign, some forms are invasive and tend to recur. CDK5 is a member of the cyclin-dependent kinase (CDK) family, and its diverse function in regulating many signaling pathways are characterized in in the development and growth of the nervous system. For instance, CDK5 controls neuronal migration and differentiation [1]. Interestingly, CDK5 activity is required for cell invasion in prostate carcinoma, glioblastoma multiforme [2], pancreatic cancer [3], medullary thyroid cancer [4]. Inhibition of CDK5 activity decreases lung cancer cell motility [5]. Liu et al. have also reported that increased CDK5 expression in patients with non-small cell lung cancer is correlated with poor prognosis [6]. Previously, we have found that active CDK5 was present in normal human pituitary tissue, was associated with $\mathrm{p} 35$, and that CDK5 activity was upregulated in diverse types of pituitary adenomas [7], suggesting CDK5 activity may play a role in pituitary tumor progression. A better understanding of cellular and molecular mechanisms mediated by CDK 5 may provide a therapeutic strategy for pituitary tumors.

Tumor angiogenesis is essential for tumor growth and invasion in pituitary adenomas $[8,9]$. Complex vascularity correlates with increased size, advanced invasiveness, poor surgical outcomes, and malignancy of prolactin pituitary adenomas [10]. Vascular endothelial 
Table 1: Clinical and pathological characteristics of the patients

\begin{tabular}{|c|c|}
\hline Variables & Patients $(n=48)$ \\
\hline Sex, F/M & $22 / 26$ \\
\hline Age, years (mean $\pm \mathrm{SD}$, range) & $39.3 \pm 10.7,14-62$ \\
\hline Macroadenoma (\%) & $23(47.9 \%)$ \\
\hline Microadenoma (\%) & $4(8.3 \%)$ \\
\hline Giant adenoma (\%) & $21(43.8)$ \\
\hline $\begin{array}{l}\text { PRL (ng/ml) } \\
\text { Grades } \\
1 \mathrm{a} \\
1 \mathrm{~b} \\
2 \mathrm{a} \\
2 \mathrm{~b} \\
\end{array}$ & $\begin{array}{l}771.6(209-5361) \\
23 \\
2 \\
20 \\
3 \\
\end{array}$ \\
\hline Mean follow-up, years (mean \pm SD, range) & $4.8 \pm 1.17,2.5-7$ \\
\hline \multicolumn{2}{|l|}{ Long-term follow-up results (\%) } \\
\hline Remission & $18(37.5 \%)$ \\
\hline Persistence & $16(33.3 \%)$ \\
\hline Recurrence & $14(29.2 \%)$ \\
\hline
\end{tabular}

F, female; M, male;

growth factor (VEGF) is critical for angiogenesis in pituitary adenomas and other neoplasms [11, 12]. Invasive pituitary prolactin adenomas exhibit an increase in vascularization than their noninvasive counterparts [13, 14]. Consistent with these findings, VEGF expression is significantly higher in invasive than noninvasive adenomas [15].

Kinase insert domain receptor (KDR), also known as Type 2 VEGF receptor (VEGFR2), mediates VEGF-induced angiogenesis under physiological and pathological conditions. KDR is a tyrosine kinase receptor, and triggers diverse intracellular responses, including the phosphoinositide 3-kinase (PI3K)/phosphorylation of protein kinase B (AKT) [16]. Abundant expression of VEGF and KDR has been identified in pituitary glands [17], and VEGF participates in the formation of vascular networks in pituitary tumors [18, 19]. Immunohistochemistry (IHC) has demonstrated that KDR is localized in vascular endothelial cells of normal and adenomatous pituitaries [10], suggesting that KDR may play a role in regulating angiogenesis. Generally, KDR signaling is initiated with tyrosine phosphorylation in the KDR cytoplasmic domain, leading to downstream effectors activation [20]. Phosphorylation at tyrosine-1212 is involved in autophosphorylation and kinase activation [21]. Mutation of both serine 1188 and serine 1191 impairs the ligand-dependent downregulation of KDR [22].

In the current study, we have sought to explore the biological functions of CDK5 and $\mathrm{p} 35$ in prolactin pituitary adenomas. We have found both p35 and p-CDK5 levels are significantly increased in invasive pituitary adenomas in comparison to noninvasive pituitary adenomas. We have identified KDR S229, located in the extracellular domain, as the only consensus phosphorylation site for
CDK5. Both inhibition and depletion of CDK5 suppress cell migration, cell invasion and KDR pSer-229 level in pituitary cells. Surface expression of S229A-KDR (nonphosphorylatable) is dramatically lower than widetype KDR (WT-KDR) in pituitary cell lines, suggesting that phosphorylation of Ser-229 (pSer-229) is required for KDR trafficking. KDR pSer-229 is upregulated in invasive pituitary adenomas and correlated with poor prognosis in patients with prolactin pituitary adenomas. Our results indicate that CDK5 mediates cell invasion through phosphorylation on KDR Ser-229 and KDR pSer-229 is a potential biomarker for pituitary adenomas.

\section{RESULTS}

\section{Expression of CDK5 and p35 in prolactin pituitary adenomas}

First, we asked whether the expression of CDK5 or p35 differs in invasive and noninvasive prolactin pituitary adenomas. We found that $\mathrm{p} 35$ expression was markedly higher in invasive than in noninvasive prolactin pituitary adenomas (Figure 1). IHC staining confirmed the upregulation of $\mathrm{p} 35$ in invasive pituitary adenomas. Immunohistochemical expression of CDK5 and p35 is shown in Figure 2. CDK5/p35-positive staining was observed predominantly in the cytoplasm. Cytoplasmic p35 staining was significantly higher in noninvasive prolactin pituitary adenomas (mean H-score: 175) than in normal pituitary tissue (mean H-score: 142), with the highest expression observed in invasive prolactin pituitary adenomas (mean H-score: 212, Figure 2A, 2B), 


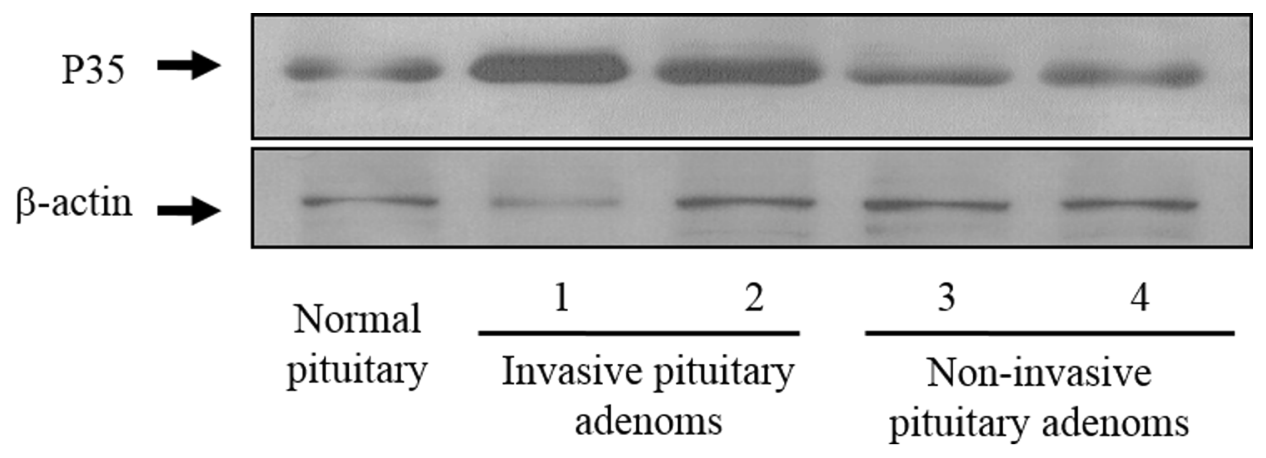

Figure 1: Representative Western blots of p35 in invasive and noninvasive prolactin pituitary adenomas. Blots were reprobed with anti- $\beta$-actin antibody to ensure equal loading.

A

Normal pituitary

Pituitary adenomas

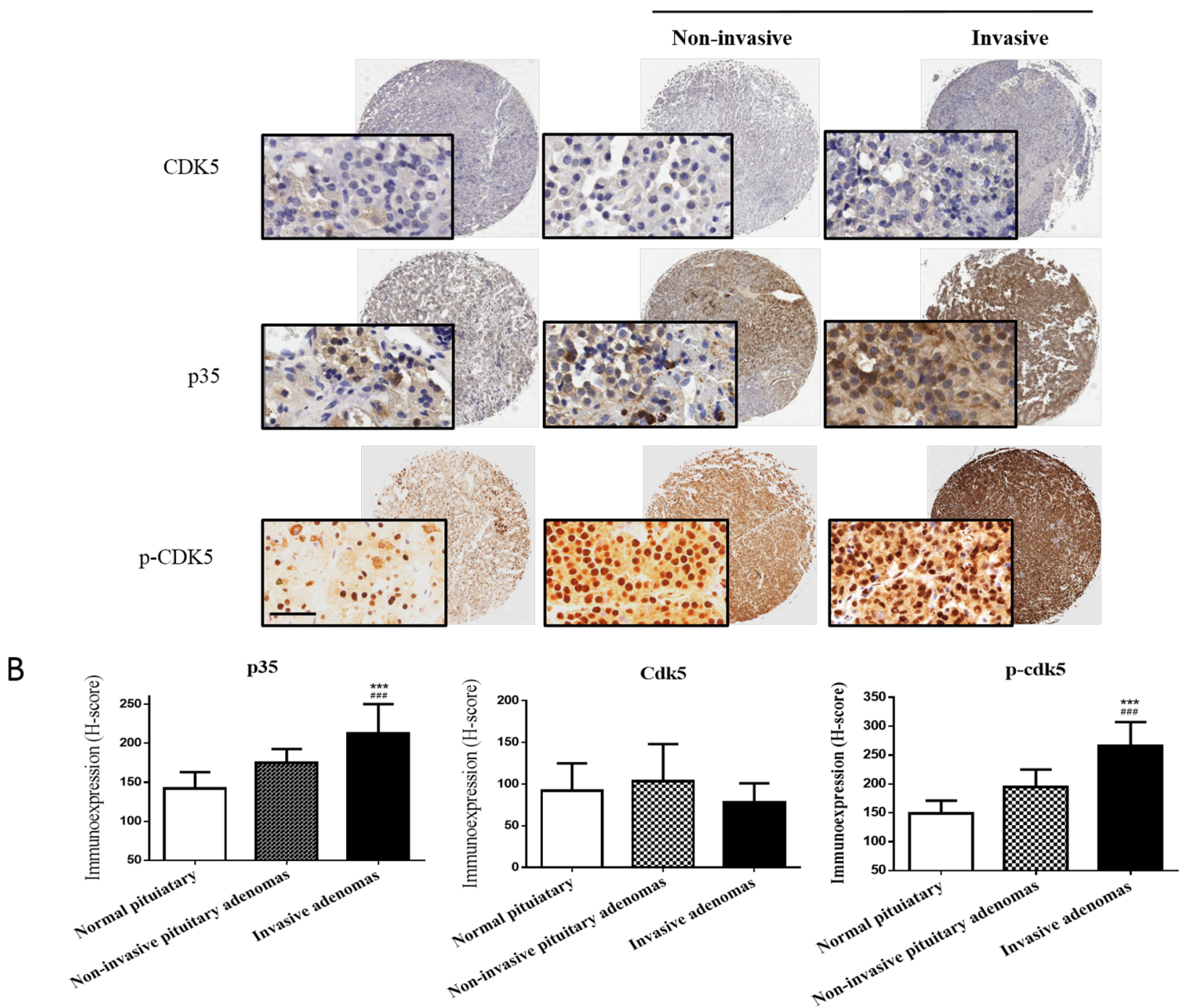

Figure 2: p35 protein and p-CDK5 levels are highly expressed in invasive pituitary adenoma tissue. A. Representative images of CDK5, p35 and p-CDK5 staining of a tissue microarray showing normal pituitary tissue with low p35/p-CDK5 expression (left panel), noninvasive pituitary adenoma with moderate p35/p-CDK5 expression (middle panel), and invasive pituitary adenoma with high p35/p-CDK5 expression levels (right panel). No significant differences in CDK5 expression level were detected. The insets show $200 \times$ magnification of the low-power images (scale bar, $50 \mu \mathrm{m}$ ). B. Mean H-scores \pm SD of CDK5, p35 and p-CDK5 staining of tissue microarrays. ${ }^{* * *} \mathrm{P}<0.001$ versus normal pituitary; \#\#\#P $<0.001$ versus noninvasive pituitary adenoma; ANOVA, followed by NewmanKeuls multiple comparison test. 
whereas no significant differences were detected in CDK5 H-scores.

To further examine CDK5 activity, we analyzed the phosphorylation levels of CDK5 in human prolactin pituitary adenomas. Expression of p-CDK5 was significantly higher in invasive (mean H-score: 266) than in noninvasive prolactin pituitary adenomas (mean H-score: 194). In addition, the p-CDK5 levels H-score was significantly higher in both invasive and noninvasive adenomas compared to normal pituitary tissues (mean H-score: 149, Figure 2A \& 2B). These results indicated that CDK5 is specifically activated in human prolactin pituitary adenomas.

\section{CDK5 inhibition suppresses GH3 cells migration and invasion}

To determine the influence of CDK5 on the migration and invasion of prolactin pituitary adenomas, we incubated GH3 rat pituitary cells with either treatments of roscovitine (a CDK 5 inhibitor) in different concentrations. Involvement of endogenous CDK5 was also tested by using short interfering RNA (siRNA) targeting the CDK5 mRNA, knockdown efficiency was verified by western blot (data not shown). The migratory ability of the GH3 cells after 48 hours of treatment was determined by the Transwell migration assay. Our data indicate that migration of $\mathrm{GH} 3$ cells was significantly reduced by roscovitine treatment (Figure $3 \mathrm{~A} \& 3 \mathrm{C}$ ) and CDK5 depletion (Figure 3B \& 3D).

Wound-healing assays showed that the gap made in the confluent monolayer of GH3 cells was closed primarily by cell migration rather than cell proliferation. The results are also calculated and presented as percentage of wound closure as a function of time. At 48 hours, approximately $40 \%$ of the initial gap had closed in vehicle-treated cells, whereas $6-29 \%$ of the gap had closed in roscovitine (Figure 3E \& 3F) or siRNA (Figure $3 \mathrm{G} \& 3 \mathrm{H})$ treated cells, indicating that roscovitine and CDK5 siRNA inhibited the cell motility. Taken together, these results suggested that roscovitine reduced gap closure by inhibiting cell motility. Our results show that cell migration and motility were significantly reduced by CDK5 knock-down. These results support the hypothesis that endogenous CDK5 plays a crucial role in GH3 cell migration and invasion.

\section{CDK5 associates and phosphorylates KDR- Ser229 in prolactin pituitary adenomas}

The pituitary contains abundant VEGF as well as KDR [23], and VEGF signaling participates in the neovascularization of pituitary tumors. Whether or not CDK5 affects the bioavailability or physiological function of KDR by phosphorylation has previously been unknown. To investigate this question, we examined whether inhibiting CDK5 activity has an effect on KDR function. To determine whether there is a direct interaction between CDK5 and KDR, we immunoprecipitated KDR from human pituitary adenoma lysates and probed with a CDK5-specific antibody. Immunoprecipitation of KDR led to coprecipitation of a detectable amount of CDK5 in noninvasive pituitary adenomas, with more intensive signaling in invasive pituitary adenomas (Figure 4A).

Phosphorylation of KDR is the key activation step for trafficking. CDK5 is a proline-directed serine/ threonine kinase, and besides an absolute requirement for proline in the +1 position, CDK 5 shows a marked preference for a basic residue in the +3 position. Its consensus phosphorylation sequence is $(\mathrm{S} / \mathrm{T}) \mathrm{PX}(\mathrm{K} / \mathrm{H} / \mathrm{R})$, where $\mathrm{X}$ can be any amino acid [24]. Using sequence analysis, we identified Ser-229 as the single putative CDK5 phosphorylation site in KDR (SPSH, located in extracellular Ig domain 3).To study Ser-229 activation, we raised a phosphospecific antibody against the pSer229 phosphopeptide. The pSer229-KDR phosphospecific antibody detected a $\sim 250 \mathrm{kDa}$ protein from wild-type green fluorescent protein (GFP)-tagged KDR expressed in GH3 cells, but it did not recognize GFP-KDR with a Ser (S) 229 to Ala (A) mutation (Figure 4B). The data indicated that pSer229-KDR phospho-antibody specifically recognized KDR phosphorylated at Ser229. To test whether CDK5 is able to phosphorylate KDR-Ser229 in vivo, we applied roscovitine (CDK5 inhibitor) and CDK5 siRNA to GH3 cells overexpressing GFP-tagged KDR. We found that both CDK5 inhibition and depletion significantly decreased the pSer229-KDR signal (Figure 4C \& 4D).

To detect whether the inhibition of Ser229KDR phosphorylation is required for CDK5-mediated migration and invasion, we cotransfected $\mathrm{GH} 3$ cells with CDK5 and WT-KDR or CDK5 with S229A-KDR (a nonphosphorylatable mutation). We found that migration (Figure 4E \& 4F) and motility (Figure 4G \& 4H) of GH3 cells were both significantly reduced in the S229A group. These data suggest that CDK5 mediates cell invasion and migration through KDR S229 phosphorylation.

\section{Ser229 is required for cell surface expression of KDR}

After confirming that CDK5 phosphorylates KDR at Ser229, we next investigated the function of S229A-KDR. We measured the total expression of WTKDR and S229A-KDR in GH3 cells. GH3 cells were transfected with GFP-tagged WT-KDR or S229A-KDR. Probing the blots with an anti-GFP antibody detected an immunoreactive band of $\sim 250 \mathrm{kDa}$ in both WT and mutant KDR-expressing cells (Figure 5A), indicating that the S229A mutation did not affect the total expression of 
A

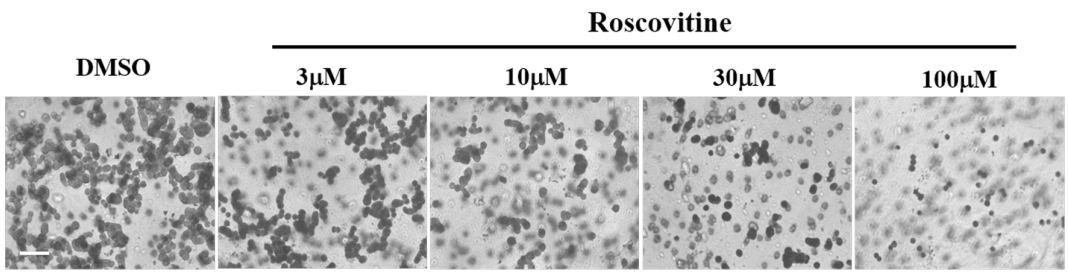

B

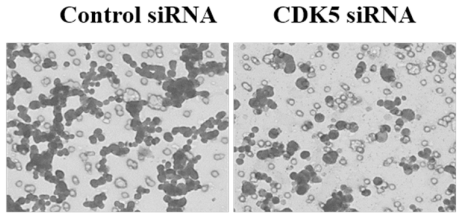

C
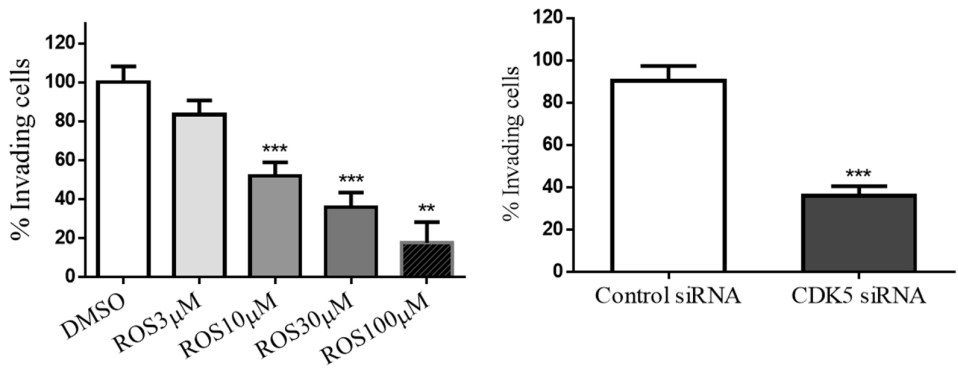

E

0

$24 \mathrm{~h}$

$48 \mathrm{~h}$

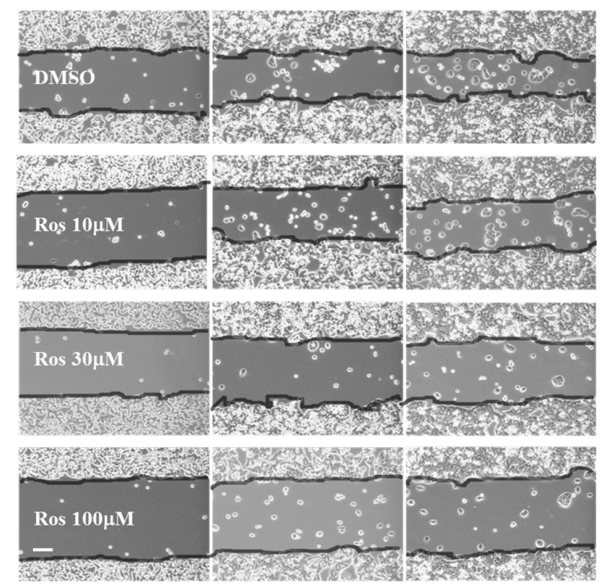

F

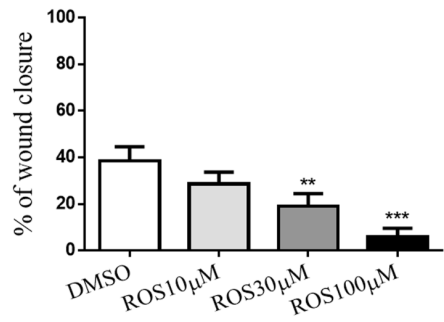

G

0

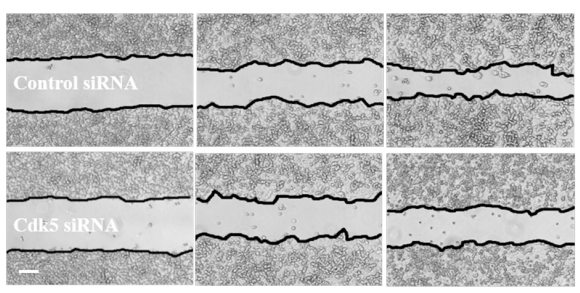

$48 \mathrm{~h}$

$\mathrm{H}$

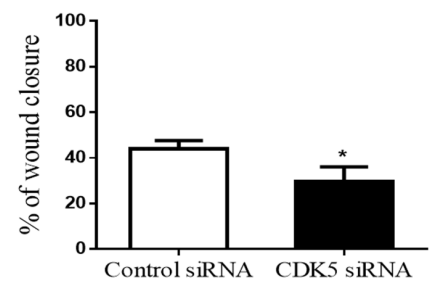

Figure 3: Both CDK5 inhibition and depletion affect the cell motility and cell migration activities of GH3 pituitary

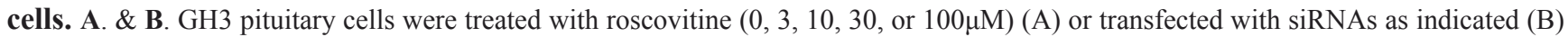
for $24 \mathrm{~h}$ and then migration was assayed in Transwell chambers (200× magnification, scale bar, $20 \mu \mathrm{m})$. C. \& D. Trend analysis of treatment dependent (C) or knock-down induced (D) invasiveness decrease of GH3 pituitary cells. E. \& G. A scratch was applied to monolayers of GH3 cells that received roscovitine treatments at indicated concentrations (E) or siRNA transfections (G), cells were observed by phasecontrast microscopy $(100 \times)$ and photographed at the indicated time points after wounding, scale bar, $200 \mu \mathrm{m}$. F \& H: Cell migration into the scratch wound after treatments $(\mathrm{F})$ or knockdown $(\mathrm{H})$ was quantified and presented as a percentage of wound healing $(\mathrm{WH} \%)$ calculated by dividing migrated distance by scratched distance. ${ }^{*} \mathrm{P}<0.05$, ${ }^{* *} \mathrm{P}<0.01,{ }^{* * *} \mathrm{P}<0.001$ versus control cultures. 
A
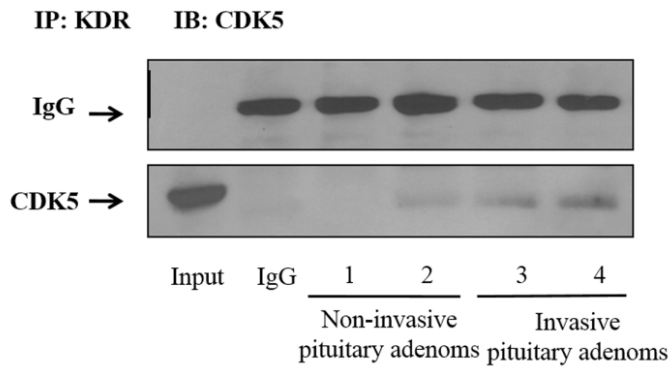

C

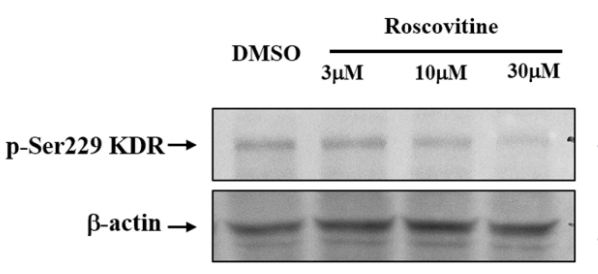

$180 \mathrm{kd}$

42kd

D

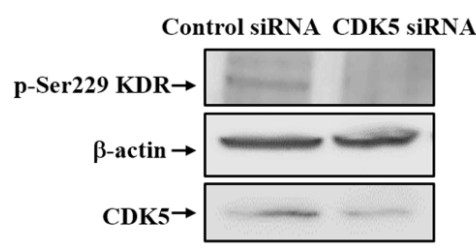

$\mathbf{E}$

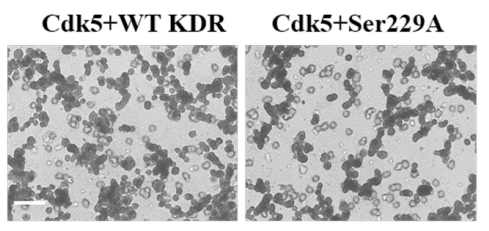

B
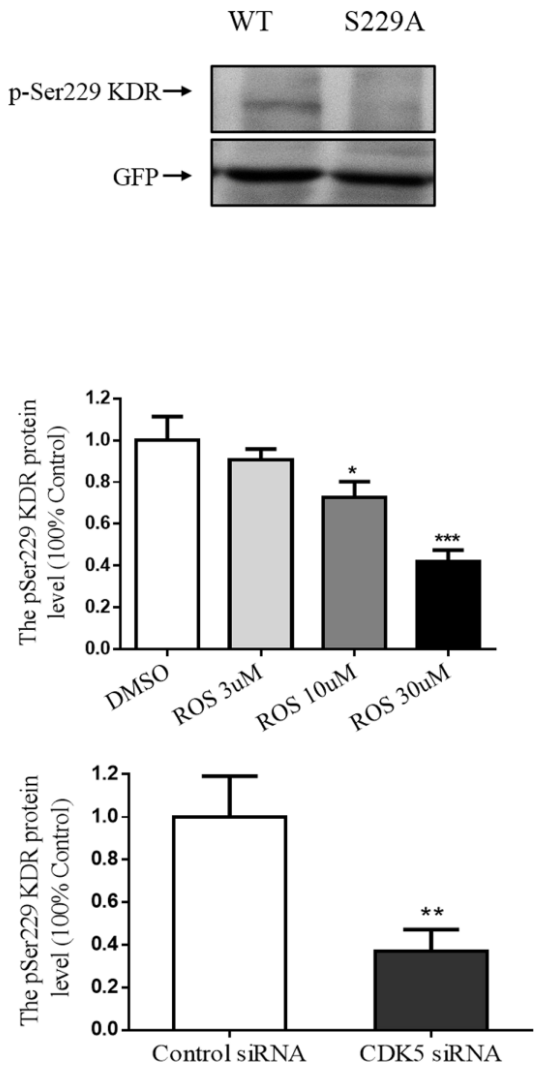

$\mathbf{F}$

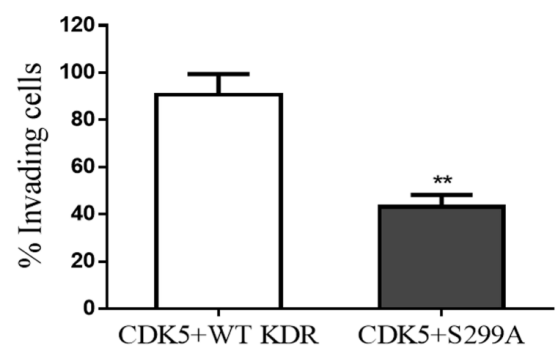

H

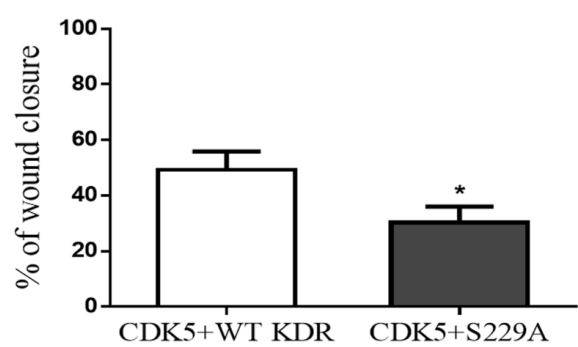

Figure 4: CDK5 regulates cell invasion via its phosphorylation of KDR at Ser-229. A. Interaction of CDK5 and KDR in prolactin pituitary adenomas. CDK5-specific antibody recognized the complex immunoprecipitated by KDR-specific antibody, but did not recognize normal IgG. B., GH3 pituitary cells were transfected with WT-KDR or S229A-KDR, and the blots were immunostained with site-specific pSer229 KDR antibody. Total cell lysates were immunoblotted with GFP and anti-actin as a loading control. C. Immunoblots (left) and densitometry analysis (right) showed KDR Ser-229 phosphorylation was reduced by roscovitine at indicated concentrations. $\beta$-actin served as a loading control. D. Immunoblots (left) and densitometry analysis (right) showed KDR Ser-229 phosphorylation was reduced by CDK 5 siRNA at indicated concentrations. $\beta$-actin served as a loading control. E. Cell migration assay of GH3 pituitary cells

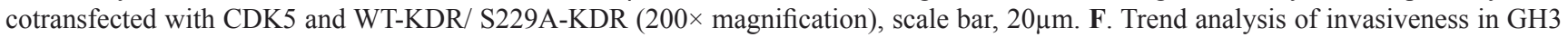
cells as indicated. G. Scratch wounds in monolayers of GH3 pituitary cell GH3 cells as indicated. Cells were observed by phase-contrast microscopy $(100 \times)$, scale bar, $200 \mu \mathrm{m}$. H: Cell migration into the scratch wound was quantified and presented as indicated. $* \mathrm{P}<0.05$, $* * \mathrm{P}<0.01, * * * \mathrm{P}<0.001$ versus control cultures (two-tailed paired $\mathrm{t}$ test within each group. Error bars indicate $\mathrm{SEM}$ ). 
KDR. Next, we used a biotinylation assay to detect the cell surface expression of KDR in GH3 cells. As shown in Figure 5A, the expression of functional S229A-KDR was significantly lower compared to WT-KDR. To further validate this result, we transfected GH3 cells with S229AKDR or WT-KDR. Confocal microscopy revealed that cells transfected with WT-KDR had greater cell surface expression of the receptor than cells containing S229AKDR (Figure 5B). S229A-KDR staining was diffuse, without cell surface expression. These data suggest that CDK5 activity contributes to the distribution of functional KDR at the cell surface.

\section{Ser229-KDR phosphorylation in human prolactin adenoma tissue}

To investigate the clinical significance of Ser229KDR phosphorylation, we carried out IHC staining of human prolactin adenoma tissue from a cohort of 48 patients with pSer229-KDR phosphoantibody. Expression of pSer229-KDR was significantly higher in invasive prolactin pituitary adenomas (mean H-score: 218) than in their noninvasive counterparts (mean H-score: 170). In addition, the pSer229-KDR H-score was significantly higher in both invasive and noninvasive adenomas than in normal pituitary tissue (mean H-score: 127) (Figure 6A). As shown in Figure 6B, the expression of pSer229KDR was significantly higher in patients with disease recurrence or persistence than in patients in remission $(\mathrm{P}<0.001)$. The prognostic value of pSer229-KDR for recurrence-free survival in prolactin pituitary adenoma patients was evaluated by comparing the patients with weak, moderate, and strong pSer229-KDR expression. According to Kaplan-Meier survival analysis, patients with high pSer229-KDR expression had a distinctly shorter recurrence-free survival time than those with weak pSer229-KDR expression $(\mathrm{P}<0.05)$ (Figure 6C). These data suggest that pSer229-KDR might serve as a prognostic biomarker to predict the outcome of prolactin adenomas.

\section{DISCUSSION}

In the present study, we have shown that $\mathrm{p} 35$ protein and p-CDK5 levels are significantly higher in invasive adenomas than in noninvasive adenomas. Inhibition and depletion of CDK5 activity suppresses cell migration and invasion of GH3 rat pituitary cells. KDR Ser-229 phosphorylation is required in CDK5 promoted cell invasion and this phosphorylation also contribute to the

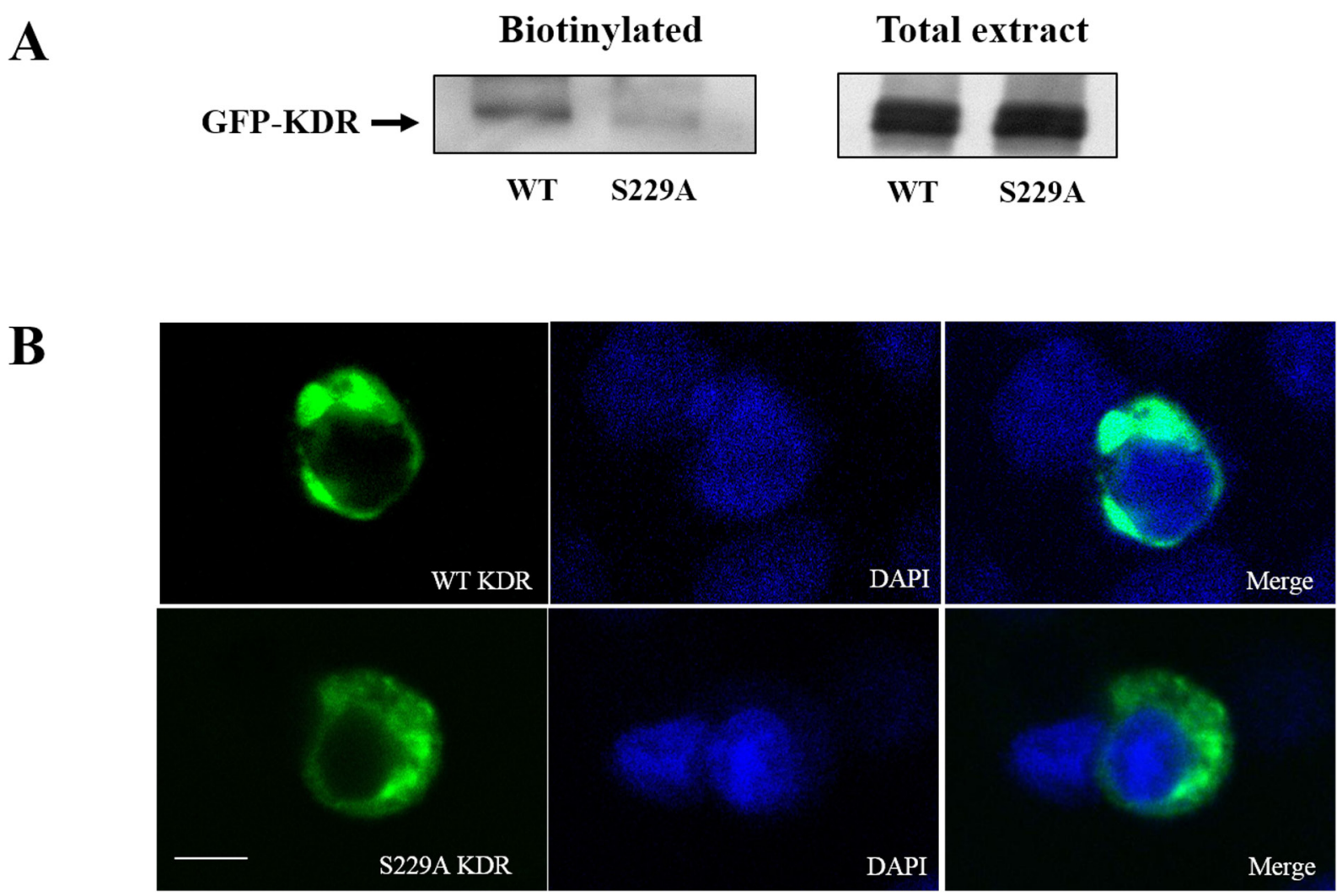

Figure 5: Ser-229 is necessary for cell surface expression of KDR. A. Surface-biotinylated KDR shows that the expression of functional S229A-KDR at the cell surface was significantly lower than expression of WT-KDR in transfected GH3 pituitary cells. B. Differences in WT-KDR and S229A-KDR distribution in GH3 pituitary cells (630×, scale bar, $10 \mu \mathrm{m})$. 
membrane trafficking of functional KDR to cell surfaces. Tissue microarray analysis of pSer229-KDR expression in 48 patients with prolactin pituitary adenomas indicated a significant correlation between high pSer229-KDR expression and poor prognosis.

Previously, CDK5 was reported to be uniquely involved in the development of the central nervous system and a key factor in neuronal migration. CDK5-dependent phosphorylation of the talin head domain at Ser425 prevents its ubiquitylation and degradation, regulating stability of cellular adhesion and cell migration [25]. Recently, CDK5 has been demonstrated to be involved in pancreatic [26], lung, and prostate [27] cancer. Although the in vitro and in vivo expression profiles of CDK5 have been investigated in several types of cancer, no published data on the expression and function of CDK5 in prolactin pituitary adenomas are available except for a report by Xie et al. [7]. The functional role of CDK5 activity in cell proliferation, migration, and invasiveness of pituitary adenoma cells remains to be elucidated. In our previous study [7], we found that active CDK5 was present in normal pituitary cells, associated with p35, and that CDK5 activity was upregulated in pituitary adenomas. CDK5 has also been shown to regulate angiogenesis and the migration of endothelial cells, and has been proposed as a target for antiangiogenic therapy [28]. Here, we have shown that both cell migration and invasion of pituitary cells were regulated by the CDK5 inhibitor roscovitine. At the concentrations applied in the current study, roscovitine would also inhibit CDK1 and CDK2; but CDK5 is the most likely target, because CDK2 expression is low in the anterior pituitary, and CDK1 is not expressed in corticotropes. By included CDK5 specific knock-down and nonphosphorylated KDR S229A mutant in this study,

A
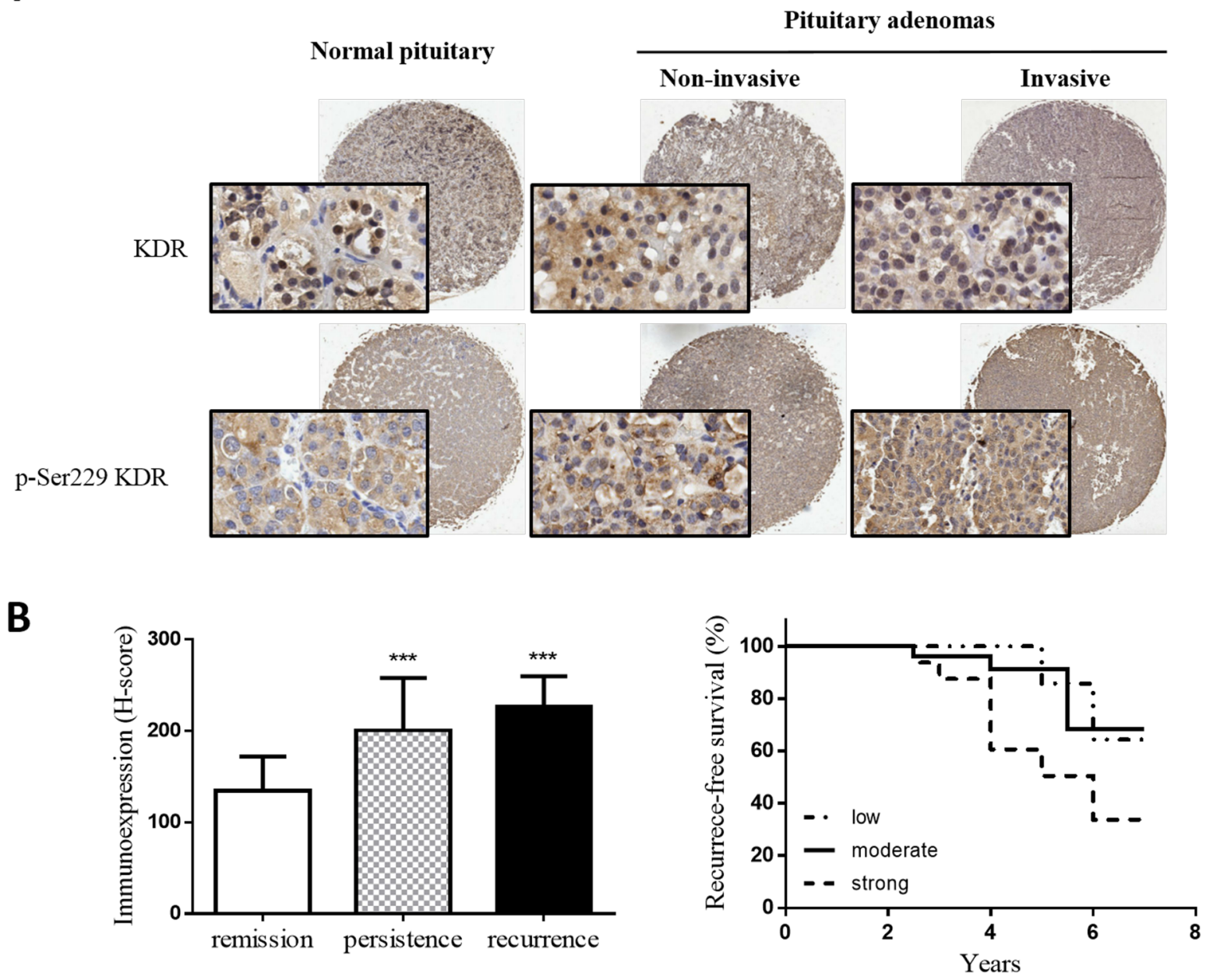

Figure 6: pSer229-KDR is highly expressed in invasive pituitary adenomas and associated with poor prognosis. A. Representative images of pSer229-KDR staining of a tissue microarray, including low pSer229-KDR level in normal pituitary tissue (left panel), moderate pSer229-KDR level in noninvasive pituitary adenoma (middle panel) and high pSer229 KDR level in invasive pituitary adenoma (right panel). Insets show 200× magnifications of the low-power images, scale bar, 50 $\mu$ m. B. Mean H-scores of pSer229-KDR expression in patients in remission and patients with persistent or recurrent disease. ${ }^{*} * * \mathrm{P}<0.001$ versus patients in remission. (ANOVA, followed by Newman-Keuls test). C: Significant differences in recurrence-free survival according to pSer229-KDR expression status in 48 patients after surgical removal of prolactin pituitary adenomas. ${ }^{*} \mathrm{P}<0.05$, by the log-rank test. 
we confirmed endogenous CDK5 regulates cell migration and invasion through the phosphorylation on KDR S229.

Human prolactin pituitary adenomas vary greatly, ranging from small indolent tumors to large invasive ones. Invasive adenomas are less sensitive to dopamine agonists (DAs) than noninvasive tumors, and they are highly vascularized in general. KDR is the primary mediator of the mitogenic, angiogenic, and permeability-enhancing effects of VEGF, and KDR intracellular signaling is regulated by phosphorylation.

The role of CDK5 in GH3 cell migration and invasiveness could be attributable to its participation in decreased KDR cell surface bioavailability. Upon VEGF binding, VEGFR2 undergoes endocytosis, triggering downstream signaling cascades. Some pathways may reach full potential at the plasma membrane level, others are VEGFR2 subcellular localization dependent. For example, VEGFR2/ERK signaling is regulated by the trafficking speed of VEGFR2/NRP1 in the cytoplasm [29]. However, the mechanisms remain unknown. Dopamine agonists can normalize prolactin secretion in 80 to $90 \%$ of microadenoma patients and $70 \%$ of macroadenoma patients. CDK5 may phosphorylate the dopamine D2 receptor and attenuate downstream signaling [30]. The data suggest that CDK5 might also regulate DA resistance and tumor growth in prolactin pituitary adenomas. The CDK5 mechanisms involved in other subtypes of pituitary adenoma will be studied in the future.

Identification of new biomarkers that predict the prognosis and therapeutic resistance of prolactin pituitary adenomas is a priority. Here, we have demonstrated that CDK5 phosphorylates KDR at Ser-229 in prolactin pituitary adenomas, a step that is required for normal cell surface expression of KDR in cell migration and invasion in vitro. Therefore, KDR phosphorylation at Ser-229 may play a critical role in invasiveness and predicting poor prognosis of prolactin pituitary adenomas. In addition, our findings showed that CDK5 inhibitors can directly or indirectly block cell migration and invasion in prolactin pituitary adenomas.

\section{MATERIALS AND METHODS}

\section{Patients}

In the present study, we retrospectively reviewed 48 patients who had undergone pituitary surgery at Beijing Tiantan Hospital between 2008 and 2012. All patients had plasma prolactin (PRL) levels $>200 \mathrm{ng} /$ $\mathrm{ml}$, and positive immunostaining for PRL. Patients with plurihormonal prolactin tumors were excluded from this study. Patients who were resistant to, or who could not tolerate dopamine agonist (DA) therapy, were included. Dopamine response was tested in 29 of the 48 patients; resistance to DA therapy was defined as previously described [31]. Medical therapy was interrupted at least 2 months before surgery. Tumor size was determined by MRI, and tumors were classified as microadenomas $(<1$ $\mathrm{cm}$ diameter), macroadenomas $(>1 \mathrm{~cm}$ and $<4 \mathrm{~cm})$, and giant adenomas $(>4 \mathrm{~cm})$. The mean postoperative followup was 4.8 years (range: $2.5-7$ years). Patients with no clinical or hormonal symptoms $(\mathrm{PRL}<30 \mathrm{ng} / \mathrm{ml})$ and no radiological evidence of disease were considered to be in remission. Persistent disease was defined as a presence of increased plasma prolactin concentration with or without a mass visible by radiology. Tumor recurrence was defined as radiological evidence of tumor regrowth. Recurrencefree survival was measured from the date of surgery to the date of tumor recurrence. Patients were censored at the date of the last neuroimaging follow-up. Normal human anterior pituitaries of people who died of non-neurological or non-endocrine diseases were obtained from a donation program. Invasive pituitary adenomas were defined as Hardy-Wilson grade IV and/or Knosp grade III and IV [32, 33]. Twenty-three patients were diagnosed with invasive prolactin pituitary adenomas and 25 with noninvasive prolactin pituitary adenomas. The Ethics Committee of Beijing Tiantan Hospital study approved the protocol, and informed consent was obtained from all patients. The patient characteristics are summarized in Table 1.

\section{Tumor samples and tissue microarray construction}

Formalin-fixed paraffin-embedded tissue blocks were sectioned and stained with hematoxylin and eosin (H\&E). Three $2.0 \mathrm{~mm}$ diameter core biopsies were selected from the paraffin-embedded tissue blocks and transferred to tissue microarrays (TMAs) using a Minicore Tissue Arrayer (Mitogen, UK). Tissue microarrays were cut into $4 \mu \mathrm{m}$ sections using a serial microtome and samples were randomly ordered and anonymized on the TMA slides. To minimize loss of antigenicity, the microarray slides were processed within 1 week of cutting.

\section{IHC techniques and antibodies}

In advance of IHC, TMA slides were stained with $H \& E$ and evaluated for quality and tumor content. TMAs were processed in a Leica BOND-III (Leica Biosystems, Germany) automated, random, and continuous-access slide staining system that simultaneously performed several IHC assays. A Bond Polymer Refine Detection system (Leica Biosystems, Germany) was used for detection of primary antibodies. Appropriate positive and negative controls were used for each antibody, and TMAs were stained for each antibody in the same run to avoid interassay variability. The immunostained slides 
were examined for expression using an Aperio AT2 digital scanner (Leica Biosystems, Germany). Primary antibodies anti-KDR (ab2349, 1/300), anti-p35 (ab66064, 1/100), and anti-CDK5 (ab40773, 1/200) were obtained from Abcam (Cambridge, MA, USA). Anti-p-CDK5 (Sc-12918, 1/100) was sourced from Santa Cruz Biotechnology (Dallas, TX, USA). Anti-pSer229-KDR ( $4 \mu \mathrm{g} / \mathrm{ml}$, Abmart), was commercially developed using standard methods by injection of specific KDR-phosphothreonine peptide AcVVL(pS)PSHGIE-amide into mice at the Abmart antibody production facility, Shanghai, China. The optimal titer of primary antibodies had been determined in previous experiments. The percentage of immunostaining and the staining intensity $(0$, negative; $1+$, weak; $2+$, moderate; and $3+$, strong) were recorded and an $\mathrm{H}$-score was calculated as follows:

$\mathrm{H}-\mathrm{score}=(\%$ cells $1+)+2(\%$ cells $2+)+3(\%$ cells $3+)$.

The maximum H-score was 300, corresponding to $100 \%$ of cells stained with strong intensity. Based on the H-score, pSer229 KDR staining in the tissue sections was categorized as weak (H-score of $\leq 100)$, moderate $(100<$ H-score $\leq 200)$, or strong $(\mathrm{H}$-score $>200)$.

\section{Cell culture}

Rat pituitary cells (GH3) were originally obtained from the China Infrastructure of Cell Line Resources (Beijing, China) and cultured at $37^{\circ} \mathrm{C}$ in $35 \mathrm{~mm}$ dishes in a humidified atmosphere of $95 \%$ air and $5 \% \mathrm{CO}_{2}$. The culture medium was Dulbecco's minimum essential medium (DMEM) with 10\% fetal bovine serum (FBS). Cultures were fed every other day. The cell lines were also genotyped to rule out cross-contamination and their morphology was regularly examined.

\section{Plasmid construction and KDR inhibitor}

The pLenti-C-mGFP-KDR (RG219851) and CDK5 siRNA (SR507441) construct were purchased from OriGene Technologies (Rockville, MD, USA). Mutant KDR (GFP-S229A-KDR) was created with the QuickChange site-directed mutagenesis kit (Stratagene; La Jolla, CA, USA). All constructs were confirmed by DNA sequencing (Shanghai Shenggong Bio, China). Roscovitine was obtained from Sigma-Aldrich (R7772; St. Louis, MO, USA).

\section{Protein extraction and Western blot analysis}

Frozen pituitary and pituitary adenoma tissues were harvested by washing with ice cold phosphate-buffered saline (PBS) three times and then scraped in ice-cold RIPA buffer (50 mM Tris, pH 7.5; 250 mM NaCl; 10 mM
EDTA; $0.5 \%$ NP-40; $1 \mu \mathrm{g} / \mathrm{mL}$ leupeptin; $1 \mathrm{mM} \mathrm{PMSF}$; and $4 \mathrm{mM} \mathrm{NaF}$ ). The homogenates were sonicated three times for $6 \mathrm{~s}$ on ice, and centrifuged at 12,000 $\mathrm{g}$ for $5 \mathrm{~min}$ at $4^{\circ} \mathrm{C}$ to yield the total protein extract in the supernatants. Protein concentration was determined with a bicinchoninic acid assay (BCA) assay kit (Pierce). Protein samples $(50 \mu \mathrm{g})$ were denatured, subjected to sodium dodecyl sulfate-polyacrylamide gel electrophoresis (SDS-PAGE) using $12 \%$ running gels, and transferred to nitrocellulose membranes. After blocking with 5\% milk powder for $1 \mathrm{~h}$ at room temperature, the membranes were incubated with primary antibody, rabbit polyclonal anti-p35 antibody (1:100; Santa Cruz Biotechnology; sc-820) and GAPDH (1:5,000; Abcam; ab6276) overnight at $4^{\circ} \mathrm{C}$.

\section{Wound-healing assay}

After GH3 cells had grown to confluence in $35 \mathrm{~mm}$ culture plates, an artificial "wound" was created using a $10 \mu \mathrm{l}$ pipette tip to scratch the cell monolayer. The wound area was inspected after 24 and $48 \mathrm{~h}$ using an inverted phase-contrast microscope with a digital camera. The wound healing speed was calculated as the percentage of the initial wound at different times until total wound closure.

\section{In vitro invasion assay}

Assays were performed using Falcon cell culture inserts ( $8 \mu \mathrm{m}$ pore size) in 24-well culture plates (BD Biosciences; Bedford, MA, USA) according to the vendor's instructions. Cell invasion was performed using Transwell chambers ( $8 \mu \mathrm{m}$ pore size; Corning Costar Corp; Cambridge, MA, USA) with Matrigel (50 $\mu \mathrm{g} /$ $\mathrm{mL}$; BD Biosciences). GH3 cells $\left(10^{5}\right.$ cells/well $)$ in 0.5 $\mathrm{ml}$ of serum-free medium containing roscovitine at the indicated concentration were seeded onto Matrigel-coated membranes in the upper chambers and incubated at $37^{\circ}$ C. The lower chambers contained the same amount of roscovitine in medium containing 10\% FBS. After 24 $\mathrm{h}$, cells that had invaded the lower chamber were fixed in $4 \%$ paraformaldehyde and stained with hematoxylin. Membranes were mounted on glass slides and observed using a phase-contrast microscope. Photographs were taken, and the number of cells in each of three randomly chosen high-power $(200 \times)$ fields was counted. All experiments were performed three times.

\section{Statistical analysis}

Results are presented as means \pm SD or medians and interquartile range (IQR), depending on data distribution. Proportions and frequencies were used for categorical variables. Comparisons between two groups were 
performed using Student's unpaired two-tailed t-test. Comparisons between three groups were performed using one-way ANOVA with Newman-Keuls test. Survival curves were calculated using the Kaplan-Meier algorithm and Log-rank (Mantel-Cox) test with GraphPad Prism 6.01. A P-value $\leq 0.05$ was considered statistically significant.

\section{ACKNOWLEDGMENTS AND FUNDING}

This work were supported by the National High Technology Research and Development Program of China (863 Program) (2014AA020610), the Research Special Fund For Public Welfare Industry of Health(201402008), National Natural Science Foundation of China (31200796), Beijing Nova Program (Z131102000413024).

\section{CONFLICTS OF INTEREST}

The authors declare no conflict of interest.

\section{REFERENCES}

1. Ghose A and Shashidhara LS. Cyclin beyond the cell cycle: new partners at the synapse. Developmental cell. 2011; 21(4):601-602.

2. Liu R, Tian B, Gearing M, Hunter S, Ye K and Mao Z. CDK5-mediated regulation of the PIKE-A-Akt pathway and glioblastoma cell invasion. Proceedings of the National Academy of Sciences of the United States of America. 2008; 105(21):7570-7575.

3. Demelash A, Rudrabhatla P, Pant HC, Wang X, Amin ND, McWhite CD, Naizhen X and Linnoila RI. Achaete-scute homologue-1 (ASH1) stimulates migration of lung cancer cells through CDK5/p35 pathway. Molecular biology of the cell. 2012; 23(15):2856-2866.

4. Lin H, Chen MC, Chiu CY, Song YM and Lin SY. CDK5 regulates STAT3 activation and cell proliferation in medullary thyroid carcinoma cells. The Journal of biological chemistry. 2007; 282(5):2776-2784.

5. Liu JL, Gu RX, Zhou XS, Zhou FZ and Wu G. Cyclindependent kinase 5 regulates the proliferation, motility and invasiveness of lung cancer cells through its effects on cytoskeletal remodeling. Molecular medicine reports. 2015; 12(3):3979-3985.

6. Liu JL, Wang XY, Huang BX, Zhu F, Zhang RG and $\mathrm{Wu}$ G. Expression of CDK5/p35 in resected patients with non-small cell lung cancer: relation to prognosis. Medical oncology (Northwood, London, England). 2011; 28(3):673678.

7. Xie W, Wang H, He Y, Li D, Gong L and Zhang Y. CDK5 and its activator P35 in normal pituitary and in pituitary adenomas: relationship to VEGF expression. International journal of biological sciences. 2014; 10 (2):192-199.
8. Di Ieva A, Grizzi F, Ceva-Grimaldi G, Aimar E, Serra S, Pisano P, Lorenzetti M, Tancioni F, Gaetani P, Crotti F, Tschabitscher M, Matula C, Rodriguez YBR (2010) The microvascular network of the pituitary gland: a model for the application of fractal geometry to the analysis of angioarchitecture and angiogenesis of brain tumors. J Neurosurg Sci 54:49-54

9. Di Ieva A, Grizzi F, Ceva-Grimaldi G, Aimar E, Serra S, Pisano P, Lorenzetti M, Tancioni F, Gaetani P, Crotti F, Tschabitscher M, Matula C and Rodriguez YBR. The microvascular network of the pituitary gland: a model for the application of fractal geometry to the analysis of angioarchitecture and angiogenesis of brain tumors. Journal of neurosurgical sciences. 2010; 54(2):49-54.

10. Onofri C, Theodoropoulou M, Losa M, Uhl E, Lange M, Arzt E, Stalla GK and Renner U. Localization of vascular endothelial growth factor (VEGF) receptors in normal and adenomatous pituitaries: detection of a non-endothelial function of VEGF in pituitary tumours. The Journal of endocrinology. 2006; 191(1):249-261.

11. Yamada S and Takada K. Angiogenesis in pituitary adenomas. Microscopy research and technique. 2003; 60(2):236-243.

12. Nomura R, Yoshida D and Teramoto A. Stromal cellderived factor-1 expression in pituitary adenoma tissues and upregulation in hypoxia. Journal of neuro-oncology. 2009; 94(2):173-181.

13. Turner HE, Nagy Z, Gatter KC, Esiri MM, Harris AL and Wass JA. Angiogenesis in pituitary adenomas - relationship to endocrine function, treatment and outcome. The Journal of endocrinology. 2000; 165(2):475-481.

14. Luque GM, Perez-Millan MI, Ornstein AM, Cristina C and Becu-Villalobos D. Inhibitory effects of antivascular endothelial growth factor strategies in experimental dopamine-resistant prolactinomas. The Journal of pharmacology and experimental therapeutics. 2011; 337(3):766-774.

15. Yarman S, Kurtulmus N, Canbolat A, Bayindir C, Bilgic $\mathrm{B}$ and Ince N. Expression of Ki-67, p53 and vascular endothelial growth factor (VEGF) concomitantly in growth hormone-secreting pituitary adenomas; which one has a role in tumor behavior? Neuro endocrinology letters. 2010; 31(6):823-828.

16. Hoeben A, Landuyt B, Highley MS, Wildiers H, Van Oosterom AT and De Bruijn EA. Vascular endothelial growth factor and angiogenesis. Pharmacol Rev. 2004; 56 (4):549-580.

17. Ochoa AL, Mitchner NA, Paynter CD, Morris RE and Ben-Jonathan N. Vascular endothelial growth factor in the rat pituitary: differential distribution and regulation by estrogen. The Journal of endocrinology. 2000; 165(2):483492.

18. Banerjee SK, Zoubine MN, Tran TM, Weston AP and Campbell DR. Overexpression of vascular endothelial growth factor164 and its co-receptor neuropilin-1 in 
estrogen-induced rat pituitary tumors and GH3 rat pituitary tumor cells. International journal of oncology. 2000; 16(2):253-260.

19. Kim K, Yoshida D and Teramoto A. Expression of hypoxiainducible factor 1alpha and vascular endothelial growth factor in pituitary adenomas. Endocrine pathology. 2005; 16(2):115-121.

20. Sakurai Y, Ohgimoto K, Kataoka Y, Yoshida N and Shibuya M. Essential role of Flk-1 (VEGF receptor 2) tyrosine residue 1173 in vasculogenesis in mice. Proceedings of the National Academy of Sciences of the United States of America. 2005; 102(4):1076-1081.

21. Meyer RD, Dayanir V, Majnoun F and Rahimi N. The presence of a single tyrosine residue at the carboxyl domain of vascular endothelial growth factor receptor-2/ FLK-1 regulates its autophosphorylation and activation of signaling molecules. The Journal of biological chemistry. 2002; 277(30):27081-27087.

22. Singh AJ, Meyer RD, Band H and Rahimi N. The carboxyl terminus of VEGFR-2 is required for PKC-mediated downregulation. Molecular biology of the cell. 2005; 16(4):21062118 .

23. Vidal S, Lloyd RV, Moya L, Scheithauer BW and Kovacs $\mathrm{K}$. Expression and distribution of vascular endothelial growth factor receptor Flk-1 in the rat pituitary. J Histochem Cytochem. 2002; 50(4):533-540.

24. Songyang Z, Lu KP, Kwon YT, Tsai LH, Filhol O, Cochet C, Brickey DA, Soderling TR, Bartleson C, Graves DJ, DeMaggio AJ, Hoekstra MF, Blenis J, Hunter T and Cantley LC. A structural basis for substrate specificities of protein Ser/Thr kinases: primary sequence preference of casein kinases I and II, NIMA, phosphorylase kinase, calmodulin-dependent kinase II, CDK5, and Erk1. Molecular and cellular biology. 1996; 16(11):6486-6493.

25. Huang C, Rajfur Z, Yousefi N, Chen Z, Jacobson K and Ginsberg MH. Talin phosphorylation by CDK5 regulates Smurfl-mediated talin head ubiquitylation and cell migration. Nature cell biology. 2009; 11(5):624-630.
26. Feldmann G, Mishra A, Hong SM, Bisht S, Strock CJ, Ball DW, Goggins M, Maitra A and Nelkin BD. Inhibiting the cyclin-dependent kinase CDK5 blocks pancreatic cancer formation and progression through the suppression of RasRal signaling. Cancer research. 2010; 70(11):4460-4469.

27. Wissing MD, Dadon T, Kim E, Piontek KB, Shim JS, Kaelber NS, Liu JO, Kachhap SK and Nelkin BD. Smallmolecule screening of PC3 prostate cancer cells identifies tilorone dihydrochloride to selectively inhibit cell growth based on cyclin-dependent kinase 5 expression. Oncology reports. 2014; 32(1):419-424.

28. Liebl J, Weitensteiner SB, Vereb G, Takacs L, Furst R, Vollmar AM and Zahler S. Cyclin-dependent kinase 5 regulates endothelial cell migration and angiogenesis. The Journal of biological chemistry. 2010; 285(46):3593235943.

29. Anthony A. Lanahan, Karlien Hermans, Filip Claes, Joanna S. Kerley-Hamilton, Zhen W. Zhuang, Frank J. Giordano, Peter Carmeliet and Michael Simons. VEGF receptor 2 endocytic trafficking regulates arterial morphogenesis. Dev Cell. 2011; 18 (5): 713-724.

30. Jeong J, Park YU, Kim DK, Lee S, Kwak Y, Lee SA, Lee H, Suh YH, Gho YS, Hwang D and Park SK. CDK5 phosphorylates dopamine D2 receptor and attenuates downstream signaling. PloS one. 2013; 8(12):e84482.

31. Delgrange E, Sassolas G, Perrin G, Jan M and Trouillas J. Clinical and histological correlations in prolactinomas, with special reference to bromocriptine resistance. Acta neurochirurgica. 2005; 147(7):751-757; discussion 757758.

32. Wilson CB. A decade of pituitary microsurgery. The Herbert Olivecrona lecture. Journal of neurosurgery. 1984; 61(5):814-833.

33. Knosp E, Steiner E, Kitz K and Matula C. Pituitary adenomas with invasion of the cavernous sinus space: a magnetic resonance imaging classification compared with surgical findings. Neurosurgery. 1993; 33(4):610-617; discussion 617-618. 